\title{
Structure, Strain Relaxation and Defects in Alkaline Earth Stannates Films
}

\section{Bharat Jalan}

\section{University of Minnesota, Minneapolis, Minnesota, United States}

Metals possessing high oxidation potential are readily oxidized, whereas those with lower potential require stronger reaction conditions. For ternary oxides such as perovskite oxides $\left(A B O_{3}\right.$, where $A$ and $B$ are elemental metals), a difference in oxidation potentials of metal $\mathrm{A}$ and $\mathrm{B}$ can make synthesis more demanding as compared to their binary oxide counterparts. For instance, if metal B has a lower oxidation potential than that of metal $\mathrm{A}$, a more severe oxidation condition may be required to achieve full oxidation of $\mathrm{B}$ in the presence of $\mathrm{A}$. Modern MBE approaches overcome these challenges to a certain extent by using high oxygen pressure, reactive gases such as ozone or by employing oxygen containing metalorganic precursors. The first two approaches often accompany undesirable consequences in MBE such as metal flux instability due to the surface oxidation, or filament oxidation, or even damage to the vacuum pumps due to high oxygen pressure. The latter approach although works for titanates and vanadates, it is not always possible to find an oxygen-containing metal precursor that is compatible with the MBE system, i.e. those with the high vapor pressure, and thermal stability. [1]

Using Sn-based compound as a model material system, we will present a detailed growth study of epitaxial, phase-pure, stoichiometric $\mathrm{BaSnO}_{3}$ and $\mathrm{SrSnO}_{3}$ films using hexamethylditin, $\left(\mathrm{CH}_{3}\right)_{6} \mathrm{Sn}_{2}(\mathrm{HMDT})$ as a tin precursor, elemental solid source for $\mathrm{Sr}$ and $\mathrm{Ba}$, and a rf plasma source for oxygen. We will demonstrate that the reactivity of tin radicals is strong enough to produce phase-pure $\mathrm{BaSnO}_{3}$ films not only with oxygen plasma but also with molecular oxygen.

Epitaxial $\mathrm{BaSnO}_{3}$ films were grown on $\mathrm{SrTiO}_{3}(001), \mathrm{LaAlO}_{3}(001)$ and LSAT (001) substrates. The substrate temperature and oxygen pressure were kept fixed at $900{ }^{\circ} \mathrm{C}$ and $5 \times 10^{-6}$ Torr respectively whereas $\mathrm{Ba} / \mathrm{Sn}$ beam equivalent pressure (BEP) ratio was varied to optimize cation stoichiometry. The unstrained lattice parameter determined using high-resolution X-ray diffraction, and the Rutherford backscattering spectroscopy (RBS) were used to optimize cation stoichiometry. Stoichiometric composition yielded an unstrained lattice parameter value of $4.116 \pm 0.001 \AA$, which is identical to that of bulk $\mathrm{BaSnO}_{3}$. Remarkably, this value was found to increase for Ba-rich films whereas the lattice parameter remains unchanged for stoichiometric and Sn-rich films. Combined with RBS and electrical transport, we discovered a MBE "growth window" where cation stoichiometry was self-regulating, i.e. for a range of $\mathrm{Ba} / \mathrm{Sn} \mathrm{BEP}$ ratio only stoichiometric films grow. Time-dependent reflection high-energy electron diffraction (RHEED) intensity oscillations were observed during film growth indicating films grew in a layer-by-layer fashion. The average spacing between $0^{\text {th }}$ order and $1^{\text {st }}$ order diffraction streaks of a timedependent RHEED pattern recorded during growth were used to investigate the evolution of strain relaxation. A critical thickness of $\sim 1 \mathrm{~nm}$ for strain relaxation was thus observed for films grown on $\mathrm{SrTiO}_{3}$. Atomic force microscopy confirmed smooth surface morphology for stoichiometric films, with a root mean square roughness value of 1-2 u.c., whereas non-stoichiometric films showed nano-crystallites on film surface indicating a correlation between surface morphology and film stoichiometry. Scanning transmission electron microscopy combined with electron energy-loss spectroscopy and energy dispersive X-ray spectroscopy confirmed the cube-on-cube epitaxy and composition Finally, we will also discuss 
temperature dependent transport properties of these films as a function of cation stoichiometry, film thicknesses and dopant concentrations. [2,3]

References

[1] A. Prakash, J. Dewey, H. Yun, J. S. Jeong, K. A. Mkhoyan and B. Jalan, "Hybrid molecular beam epitaxy growth for stoichiometric BaSnO3”, J. Vac. Sci. Technol. A 33, 060608 (2015)

[2] A. Prakash, P. Xu, A. Faghaninia, S. Shukla, J. W. Ager III, C. S. Lo, and B. Jalan, "Wide Bandgap $\mathrm{BaSnO}_{3}$ Films with Room Temperature Conductivity Exceeding $10^{4} \mathrm{Scm}^{-1}$ " Nat. Comm. 8, 15167 (2017) [3] A. Prakash, P. Xu, X. Wu, G. Haugstad, X. Wang, and B. Jalan, "Adsorption-Controlled Growth and the Influence of Stoichiometry on Electronic Transport of Hybrid Molecular Beam Epitaxy-Grown $\mathrm{BaSnO}_{3}$ Films" J. Mater. Chem. C, 5, 5730 (2017) 